\title{
Horizontal Compensatory Eye Movements in Goldfish (Carassius auratus)
}

\section{The Normal Animal}

\author{
Stephen S. Easter, Jr., Pamela R. Johns, and Donald Heckenlively \\ Department of Zoology, University of Michigan, Ann Arbor, Michigan, USA
}

Received February 8, 1974

Summary. 1. Horizontal eye and head movements made by freely swimming goldfish have been recorded cinematographically and analyzed frame-by-frame.

2. Most horizontal eye movements occur during turns. A binocular saccade precedes a turn, and the eyes counterrotate relative to the head as the turn progresses, thus keeping a nearly constant orientation in space. The subsequent saccades reset the eyes from an intermediate position to an extreme one.

3. Both the saccades and the compensatory movements are generally unequal in the two eyes. The rotation by the eye on the outside of a turn exceeds that of the inner one, which undercompensates.

4. The compensation factor, $\mathrm{CF}$, defined as the ratio of (mean binocular rotation relative to the head)/(head rotation), is variable from turn to turn, but averages $0.95 \pm 0.10$ (mean \pm 2 S.E.M.).

5 . The slight undercompensation by the inside eye, when coupled with forward movement by the fish, results in relative visual stabilization of a region of space some tens of centimeters or less lateral to the animal. This stabilized region changes with each saccade.

\section{Introduction}

Animals capable of moving head and eyes usually coordinate the two reciprocally, so that as the head moves one way, the eyes move the other, keeping the retinal images relatively stable. Walls (1962) suggested that this function of image stabilization propelled the evolution of eye movements, for it seemed that a relatively stable image would be more susceptible to neural analysis than a moving one. Visual performance would therefore be improved and the animal kept better informed of its environment by the ability to move its eyes in a way that compensated the head movement.

If the head rotated about the same axis as one eye, it would be possible to stabilize the image of the entire visual environment on the retina of that eye, simply by making the eye rotate by an amount equal and opposite to the head. But, in fact, the head does not usually rotate concentrically with an eye, or even about any axis through the head. Far more commonly, rotation is coupled with translation of the head which puts the axis of rotation outside the body. Under these conditions, 
it is geometrically impossible to stabilize the entire visual environment of either eye. If the ocular rotation exactly cancels that of the head, then a plane at infinity will be stabilized, and all stationary objects will seem to move in a direction opposite to the animal's translation. Conversely, if near objects are stabilized, then distant objects will appear to move in the same direction as the animal and very near objects in the opposite sense. This is the well-known phenomenon of motion parallax. It is experienced continually by mobile animals with lateral eyes, but very little attention has been paid to how these animals respond. Do they compensate "perfectly;" that is, rotate their eyes with respect to the head through an angle equal and opposite to that of the head, and thus maintain an unchanging orientation in space? Or do they compensate "imperfectly," in such a way as to stabilize on the retina an image of some region short of infinity? (Note that the adjective, "perfect," refers only to the accuracy with which the eyes maintain their position in space, and does not imply visual utility of the accuracy.)

Two published reports have dealt with this question. In the first, Harris (1965) worked on the dogfish, Squalus acanthias L. and found that it undercompensated by a considerable amount. In the second report, Hermann and Constantine (1971) observed several species of teleost, including goldfish, and concluded that compensation was perfect. But the accuracy of their measurements of ocular orientation left considerable room for doubt; for example, in their Fig. 6C, the "average of differences between two independent scorings" of the same picture was 5-10 degrees.

In view of the fact that "perfect" compensation is maladaptive, by the criterion of Walls (1962), we have undertaken a reinvestigation of horizontal compensatory eye movements in the goldfish. Our measurements employed rigid pointers attached to the corneas (Harris, 1965; Easter, 1971); these sharply visible stalks can be measured with greater accuracy than Hermann and Constantine (1971) claimed for their observations. Our results are presented in two papers. In this one we describe the horizontal compensatory eye movements of normal animals, discuss the visual consequences of our findings, and attempt to interpret these consequences according to our understanding of the goldfish visual system. In the second paper (Easter and Johns, 1974), we report an experimental attempt to determine the sources of information used. by the animal in making these movements.

\section{Methods Data Acquisition}

Goldfish, Carassius auratus, $15-25 \mathrm{~cm}$ in length, were obtained from Ozark Fisheries, Inc., Stoutland, Mo. A black stalk was attached by suction normal 
( \pm 5 degrees) to each cornea. The stalk ( $2 \mathrm{~mm}$ wide, $11 \mathrm{~mm}$ long), was made of polyethylene tubing (PE 240), sealed at one end, flared at the other, and filled with black ink. It partially blocked the pupil, but did not interfere very much with vision, as fish with these stalks on their eyes made optokinetic pursuit movements (Easter, 1972).

The fish swam in a circular pool of water $10 \mathrm{~cm}$ deep, one meter in diameter. Alternate black/white vertical stripes (reflectance ratio 1/9, determined photometrically) lined the sides of the tank. They were $2.5 \mathrm{~cm}$ wide, $10 \mathrm{~cm}$ high, and therefore subtended about $3^{\circ}$ horizontally and $12^{\circ}$ vertically from the center of the tank. The visual acuity of goldfish, measured behaviorally, is about 1.6 degrees (Rowley, 1934; see Charman and Tucker, 1973), so the stripes should have been sharply visible. A $16 \mathrm{~mm}$ cinema camera (Bolex H-16) with an $86 \mathrm{~mm}$ focal length lens was mounted on a tripod and filmed the pool from a distance of about three meters, off a front surface mirror mounted above the pool. On the white floor of the pool, a rectangle, $24 \times 16 \mathrm{~cm}$, was drawn in the center. The rectangle nearly filled the eine-frame, and the camera was turned on whenever the freely-swimming fish entered this area.

\section{Data Analysis}

Individual frames were viewed on a rear projection screen marked with a reticule. The orientations of the eyes were obtained by measuring the angles formed by each stalk and a horizontal reference line on the reticule, as shown in the inset of Fig. 2. Independent measurements of the stalk angles in a given frame varied by no more than 2 degrees. The head's orientation was estimated by positioning a straight line to bisect the head parallel to its longitudinal axis. This measurement was less exact; its variability was typically 4 degrees or less. In nearly all cases, only one determination was made of each set of the three angles, which will hereafter be abbreviated as LE, RE, and $\mathrm{H}$.

By convention, angles were recorded with the counterclockwise direction taken as positive, clockwise, negative. Angles measured as described in the preceding paragraph are considered to be in laboratory coordinates; that is, relative to the rest of the world. Occasionally, eye positions relative to the head may be used. When they are, they are so labeled.

In some cases, all frames were measured, and the complete time course of the activity could then be plotted, as in Fig. 2. More commonly, however, we needed only to know the extent of a movement such as a saccade or an intersaccadic drift. In these cases we measured more selectively, according to the following plan. The film was projected at normal speed until a saccade was detected, whereupon if was stopped, and the three angles (LE, RE, and $\mathrm{H}$ ) were recorded. These were taken as post-saccadic values. Then the film was run backwards by a number of frames which corresponded to about $1 / 4 \mathrm{sec}$ in real time. This one-quarter second interval was chosen because it always included the entire saccade and any minor adjustments. The three pre-saccadic measurements were made, and the film continued forward. Subtraction of appropriate pairs of values give the travel during or between saccades.

The data were analyzed statistically at the University of Michigan Computing Center, using the MIDAS Program developed at the Statistical Research Laboratory of the University.

\section{Results}

The Turn

A typical turn is illustrated in Fig. 1, made by tracing the projected images of eye stalks and body. The animal entered the picture from the 


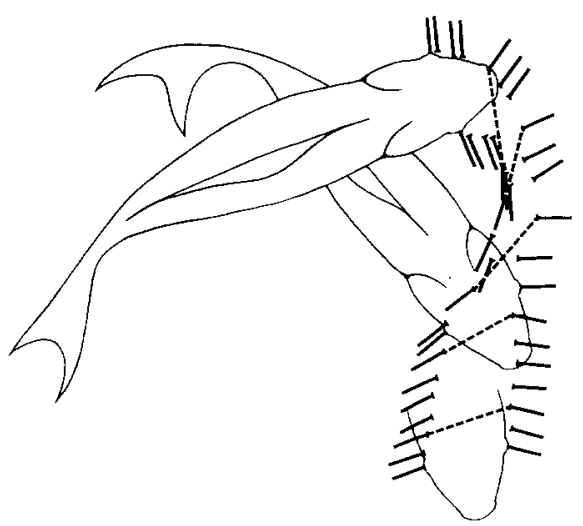

Fig. 1. This shows a dorsal view of successive positions of the eye stalks and, less frequently, the head and body of one fish during a turn to the right. The drawing was made from the projected images of a filmed record. The first position is at the top, with the entire body and both eye stalks drawn in. Throughout the rest of the turn, the eye stalks are shown at intervals of $12 / 64 \mathrm{sec}$. The animal made five saccades. The first pair of eye stalk positions following each saccade are connected by the dashed lines

left, swam straight ahead, and then turned to its right. The sequence was filmed at 64 frames per second, but only every twelfth frame is figured here. The dashed lines connect the bases of the stalks just after each of five saccades which the animal made.

Fig. 2 illustrates this same turn more completely, as the eye stalks' positions are given every frame, and the head, every other. The upper graph, Fig. 2a, shows the three angles in laboratory coordinates. Beneath, in Fig. $2 b$, the same data are presented, but this time the ocular orientations are given with respect to the head.

The first four positions in Fig. 1 and the first half second in Fig. 2 show that the orientations of the eyes remained nearly fixed in space when the animal moved straight ahead. Just prior to the head's turn, a binocular saccade abruptly shifted the eyes clockwise from their roughly symmetrical positions. Shortly after completion of the saccade, the head began to turn, and as it did, the eyes maintained their new orientations in space to within a few degrees. As turning continued, the eyes rotated back toward their starting positions (see Fig. 2b), a new pair of saccades occurred, and the nystagmus was repeated.

These graphs show that during an active turn the eyes moved smoothly toward intermediate positions from extremes and the saccades reset the eyes at extreme positions in the direction of the turn. This is similar to optokinetic nystagmus (Easter, 1972) and contrary to the 

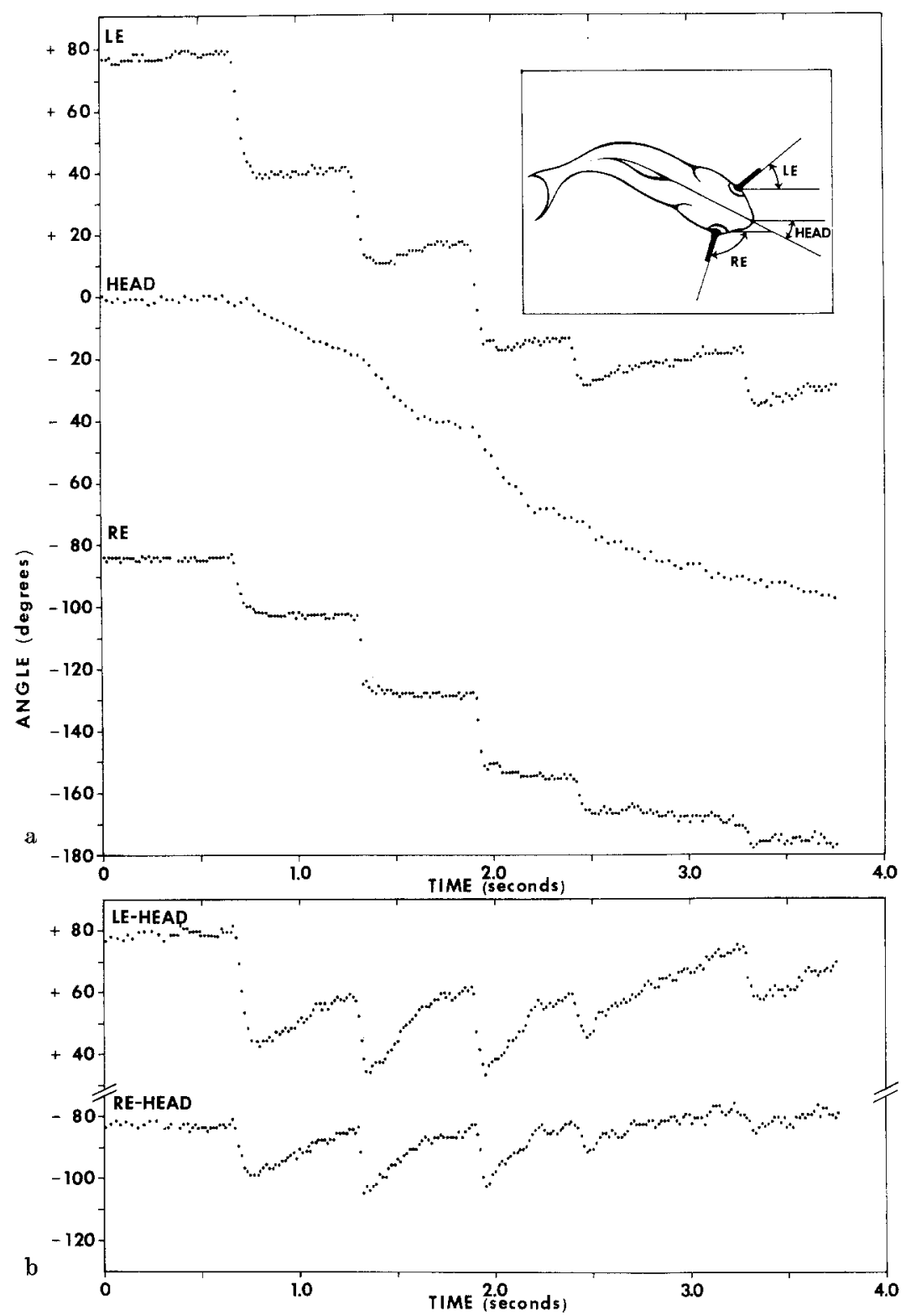

Fig. $2 a$ and $b$. This represents the same turn shown in Fig. 1. The inset gives the conventions. In all cases, clockwise movements viewed from above are taken as negative, counterclockwise as positive. Filming rate: 64 frames/sec. a) The top three traces $(L E, H, R E)$ illustrate the head and eye movements in laboratory coordinates. b) The lower two traces $(L E-H, R E-H)$ show the ocular rotations with respect to the head 


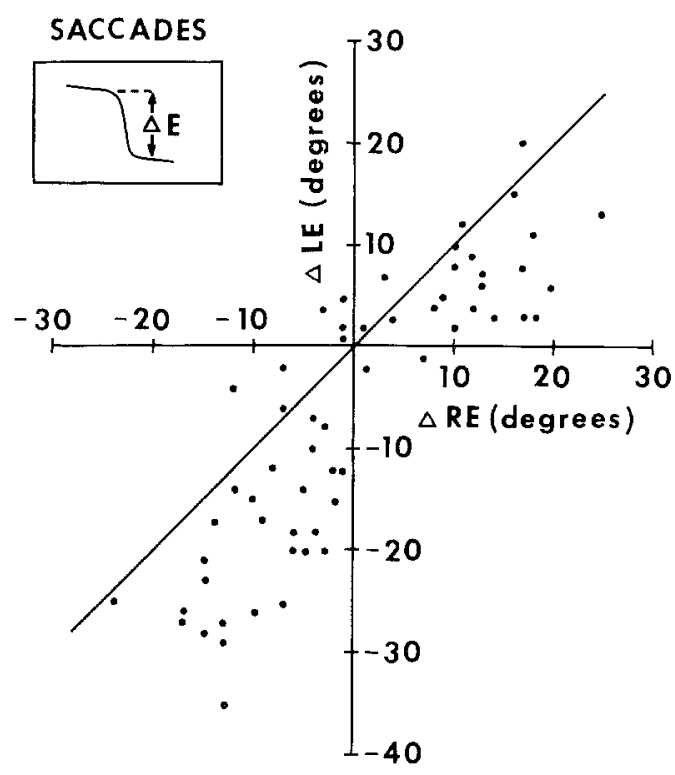

Fig. 3. This scatter diagram shows the simultaneous horizontal saccades by the left and right eyes plotted on the ordinate and abscissa, respectively. The diagonal has unit slope. The data are from one fish. The conventions are the same as in

Fig. 2

descriptions of nystagmus which have appeared in the literature (e.g. Walls, 1962; Lowenstein, 1971), where it was claimed that fish's eyes move smoothly toward an anatomically-determined limit, and then reset toward an intermediate position.

\section{Saccades}

Before going into the accuracy of the compensatory slow movements, a point should be made about the saccades. It has been shown that restrained goldfish generally make binocular saccades which are unequal (Easter, 1971), and such eye movements are termed "disconjugate." Fig. 2 shows that the same was true for the freely-swimming animal. The first and fifth saccades were particularly notable in this respect, as the left eye moved about 20 degrees farther than the right, in both cases.

In order to determine if disconjugate saccades were common, the entire filmed record was analyzed selectively, taking only pre- and post-saccadic values. The data obtained from these measurements are given in Fig. 3 ; a scatter diagram in which a point's position corresponds to the simultaneous movements made by the two eyes. Conjugate 


\section{SUMMARY OF RESULTS}

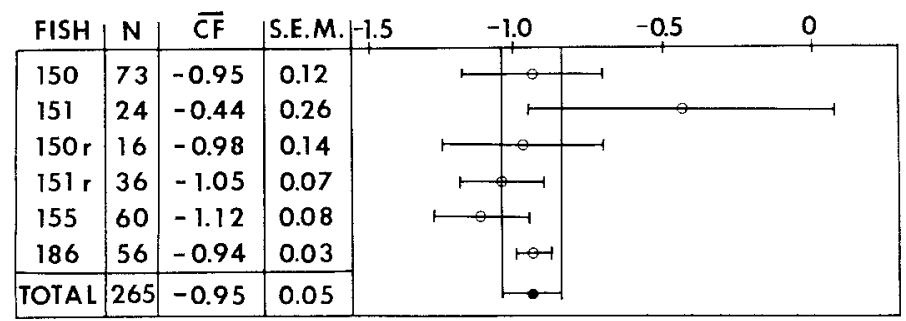

Fig. 4. This summarizes the results obtained from four normal fish. The first column on the left identifies the animal, the second gives the number of intersaccadic intervals measured. $\overline{C F}$ is the mean compensation factor, defined in the text, and S.E.M. the standard error of this mean. The symbols to the right show $\overline{C F} \pm 2$ S.E.M. for the animal in that row. The open circles are for individuals; the filled circle and bars (bottom row) gives the same statistie for the pooled data. The $95 \%$ confidence limits for the entire sample are extended upwards for purposes of comparison

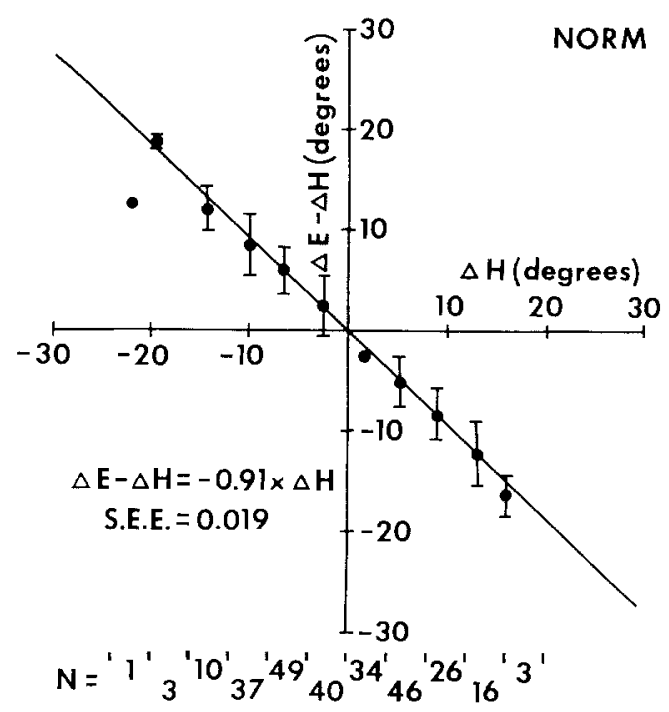

Fig. 5. This shows the ocular rotation relative to the head (ordinate) vs. the simultaneous rotation by the head, in laboratory coordinates (abscissa). The data were pooled in 4 degree-wide bins and the means and standard deviations computed. The filled circles give the two means within each bin; the bars indicate \pm 1 standard deviation. The least squares regression line is drawn, and its equation given, along with the standard error of the estimate of the regression coefficient. At the very bottom are given the numbers of measurements in each bin 
saccades would fall on the diagonal of unity slope. Inspection shows that most saccades fell below this level, and were therefore convergent, just as Fig. 2 suggested. Nearly all these eye movements occurred during or just prior to a turn, so it follows that most turns were preceded and accompanied by saccades in which the nasad rotation of the outer eye (e.g. the left eye in a right turn) exceeded the temporad rotation by the inner eye. As a consequence, the region of binocular overlap of the visual fields, which lies ahead of the animal during linear swimming, is shifted, during a turn, in the direction of the turn.

\section{Accuracy of Compensation}

Individual compensatory movements were suprisingly inaccurate; during some intersaccadic intervals, the eye moved by as much as 10 degrees in laboratory coordinates. We guessed that this failure to cancel the head movement might have resulted from excessive velocities of head rotation, which sometimes reached 70 degrees/sec. This was rejected when the data were reanalysed in terms of velocity rather than position, and we found that poor compensation was independent of velocity of head rotation.

The average accuracy of binocular compensation was obtained by pooling the results of four fish, and a summary of these data appear in Fig. 4. In the column on the left, the individual fish are identified by number and condition. For instance, 150 and $150 \mathrm{r}$ are the same animal, but the absence of suffix indicates that the data were taken when the animal was normal with no known history of neural lesions. The animal was subsequently blinded and then regenerated vision. The suffix, $r$, denotes that vision has regenerated. (See Easter and Johns, 1974, for details.) The second column gives the number of measurements in the data set for that row. The third column gives the mean of the compensation factor, CF, computed from individual $i$ th turns:

$$
\overline{\mathrm{CF}}=\frac{1}{N} \sum_{i=1}^{N} \frac{\Delta E_{i}-\Delta H_{i}}{\Delta H_{i}}
$$

in which

$$
\begin{aligned}
\Delta E_{i} & =\frac{\left(\Delta L E_{i}+\Delta R E_{i}\right)}{2} . \\
N & =\text { number of samples. }
\end{aligned}
$$

The fourth column gives the standard error of this mean. The graph, to the extreme right, displays $\overline{\mathrm{CF}} \pm 2$ S.E.M., the $95 \%$ confidence limits of the estimate. In subsequent discussion, the $\overline{\mathrm{CF}}$ will be given as $\overline{\mathrm{CF}} \pm 2$ S.E.M. 


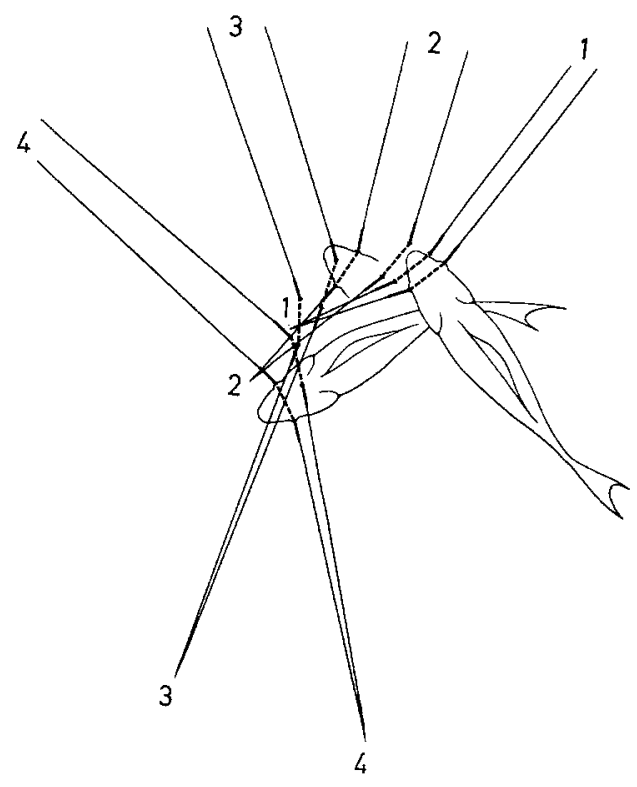

Fig. 6. This shows successive positions of the eye stalks, head, and body of one fish during a turn to the left. Each stalk is shown at eight positions, chosen to be the beginning or the end of an intersaccadic interval. The dashed lines connect simultaneous stalk positions. The numbers indicate successive intersaccadic intervals. During each, the outside (right) eye compensated nearly perfectly and therefore stabilized a plane at infinity. The inside (left) eye undercompensated and stabilized four successive points, indicated by the numbers and the intersections to the animal's left

All six examples gave individual $95 \%$ confidence limits which overlapped with each other and with the $95 \%$ confidence limit of the pooled data. The $\overline{\mathrm{CF}}$ for the group as a whole was $0.95 \pm 0.10$.

The $\overline{\mathrm{CF}}$ was roughly constant for turns of all sizes, as Fig. 5 demonstrates. In this case, the data from all four animals were pooled, assigned to 4-degree-wide bins of $\Delta H$, and the means and standard deviations were computed for each bin. The spots and vertical bars give the mean \pm the standard deviation.

The line through the data is the least squares linear regression through the origin. The regression equation is also given on the graph along with the standard error of the estimate. The regression coefficient is close to, but not identical with, $\overline{\mathrm{CF}}$. The good fit of the regression line to the data indicates that ocular rotation depended linearly on head rotation, regardless of turn size. 


\section{Disconjugacy of Compensatory Movements}

It has been pointed out above that compensation was quite variable from turn to turn, even in the same animal. There was, however, a tendency for the outer eye to counterrotate more than the inner one, which usually undercompensated. The mean value of the ratio (rotation of outside eye relative to the head)/(rotation of inside eye relative to the head) was 1.31. This disconjugacy has an interesting visual consequence, as shown in Fig. 6. The positions of the stalks before and after each saccade have been drawn in, with intervening positions omitted. Certain pairs can be seen to intersect on the inside of the turn. In every case, these are the positions observed at the beginning and end of one intersaccadic interval, which implies that, during that period, the stalk pointed at the intersection. Since the stalk also pointed through the line of sight, it follows that the image of the point of intersection was stabilized on the center of the retina. As the illustration shows, this lay some centimeters lateral to the fish, and changed with each saccade. This sort of stepwise stabilization of near objects was first observed by Harris (1965) in his studies of the dogfish. It will be developed further in the Discussion.

A very similar analysis leads to the conclusion that overcompensation by the outside eye results in stabilization on that retina of a region of space on that side of the animal. An example of overcompensation by the outer eye was shown in Fig. 2. Examination of many turns revealed that the stabilized regions on the outer side of the turn usually lay many tens of centimeters, and sometimes, meters, distant.

In closing, it is emphasized that the under- and overcompensation by the inside and outside eyes were not observed during every turn, but were average behaviors.

\section{Discussion}

The advantages of perfect compensation are questionable since stabilization of a plane at infinity assures that the retinal images of near objects will move. This consequenes is particularly troublesome for aquatic animals, since sighting distances in natural bodies of water are typically measured in meters or fractions thereof (Duntley, 1963). Although Fig. 4 showed that average binocular compensation was nearly perfect, as Hermann and Constantine (1971) had originally concluded, examination of the behavior of the eyes individually showed that compensation was quite imperfact, and systematically so, as illustrated in Fig. 6.

The visual consequences of undercompensation by the inner eye have already been introduced. They will now be developed further. Only the two-dimensional case in the horizontal will be treated. 
a
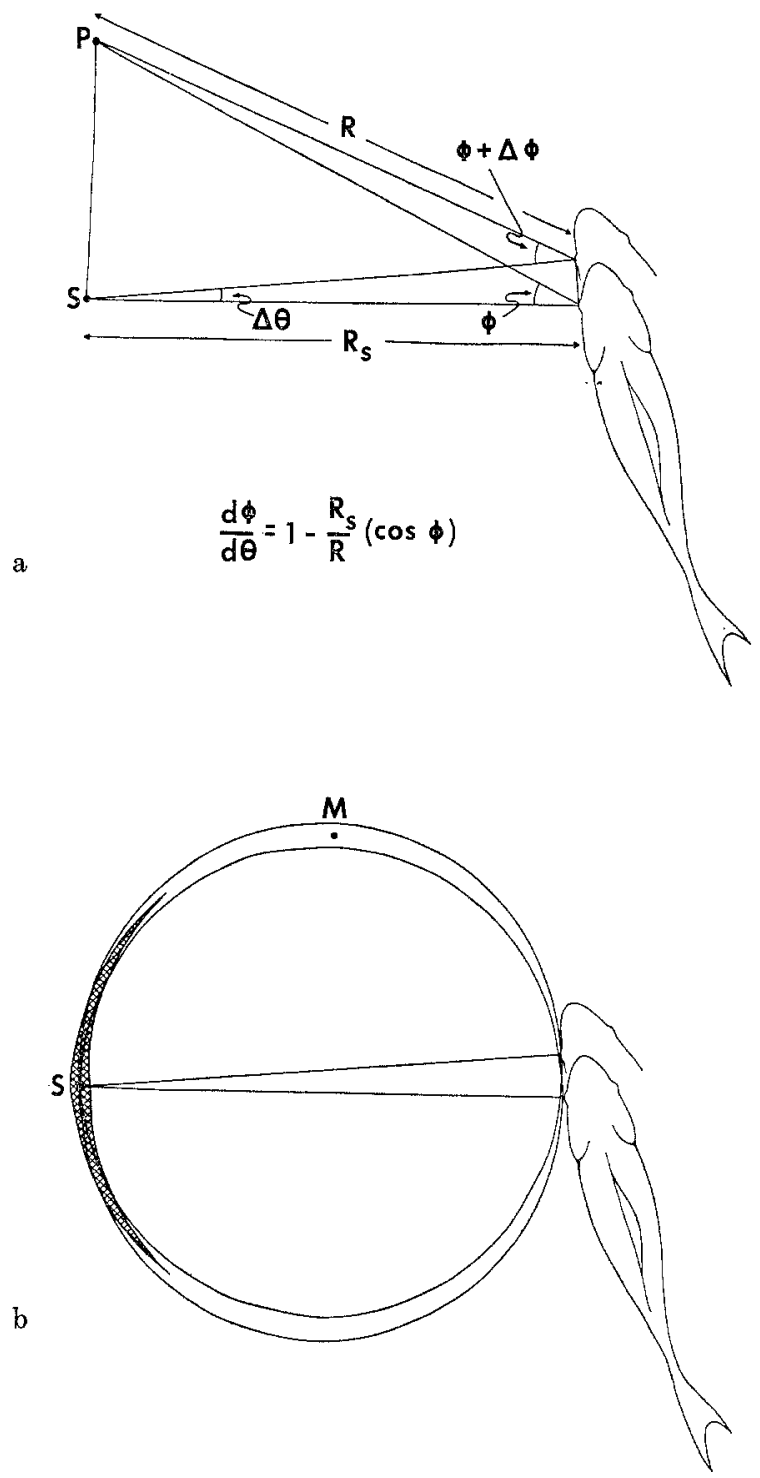

Fig. 7. a) The top drawing schematizes a fish turning left stabilizing the point, $S$, a distance $R_{S}$ away. The position of any point on the retina is given by $\phi$, referred to the retinal locus of the image $S . P$ is one such point, a distance $R$ away. As the animal turns, and the eye rotates through $\Delta \theta$, the retinal locus of $P$ shifts by $\Delta \phi$. The expression for $d \phi / d \theta$ is given. b) The lower drawing gives a similar turn, and shows two circles, both passing through $S$ and each tangent to the eye at the beginning or the end of the turn. $M$ is a point whose retinal image oscillates during the turn. Within the shaded region, retinal image velocities will be below the animal's threshold of detection. See text for details 
Consider a small turnlet during which the eye pivots about the stable point, $S$, in Fig. 7a. This direction is arbitrarily taken as $\phi=0$. $S$ is imaged at the same point on the retina throughout the turnlet, but in general, the retinal images conjugate with other points in space move during this time. Consider $P$, an arbitrary point specified by $(R, \phi)$, the two polar coordinates. At the beginning of the turnlet, $P$ was imaged along the ray $\phi$, but at the end, following translation of the eye through $R_{S} \Delta \theta, P$ is now on the ray $\phi+\Delta \phi$.

The problem is to derive a general expression for $d \phi / d \theta$, the movement of retinal images during turnlets. This is done by considering the triangle formed by the points $P, S$, and the eye. When the angles and sides are expressed in terms of one another, then the derivative in question is given by:

Substitution of

$$
\frac{d \phi}{d \theta}=1-\left[\frac{R_{S}}{R} \cos \phi\right]
$$

$$
\frac{d \phi}{d \theta}=0
$$

into the equation permits calculation of the locus of points for which the image velocity is zero at any instant during a turn. It is a circle tangent to the eye, with its center passing through the point with the polar coordinates $\left(R_{S} / 2,0\right)$. All stationary points within this circle appear to move in the temporal direction, while those outside the circle move nasad. Note that this locus of points is valid only instantaneously, for after a brief time, when the eye has pivoted through $\Delta \theta$ about $S$, there is a new circle defined by the diameter drawn from $S$ to the new position of the eye. Two circles, corresponding to the zero velocity contours at the beginning and the end of a ten degree turn, are shown in Fig. 7. Throughout the turn, $S$ was stable, but point $M$, for instance, was initially sensed as moving forward, then ceased moving and began moving in the opposite direction as the new circle moved to enclose it. Analogous to and from movements are sensed throughout the entire crescent in the nasal field; reverse oscillations occur in the temporal crescent.

It is intuitively obvious that the apparent movement of objects near $S$ will be very small. Since the minimum image velocity that the goldfish retina can sense is known to be about 0.5 degrees/second (Easter, 1972; Wartzok and Marks, 1973), it is probably fair to say that retinal images moving more slowly than that can be considered stationary. For a typical turning velocity of 25 degrees/second, $d \phi / d t$ would be less than 0.5 degrees/sec within the contours given by $d \phi / d \theta=$ \pm 0.02 . These contours are approximately given by two circles concentric with the one for zero velocity, with radii $0.49 R_{S}$ and $0.51 R_{S}$. The area enclosed by the intersections of these four circles (one pair 
for both the initial and final positions) is given by the shaded region in Fig. $7 \mathrm{~b}$. In three dimensions, the point, $S$, projects vertically as a line. We conclude that the undercompensation results in effective visual stabilization of the contents of a high, broad, and not very deep shell, concave toward the animal.

Thus, we have probed the hypothesis that compensatory eye movements aid visual stabilization of the environment. We have added new measurements and theory which show what parts of the visual environment are stabilized. It remains now to consider how the fish manages to do this, and that is the subject of the following paper (Easter and Johns, 1974).

This work was supported by grant EY-00168 from the National Eye Institute of the United State Public Health Service.

We thank Drs. M. Alpern and R. Knighton for useful critical discussions, C. Gans for the extended loan of the motion picture analyzer, Mr. L. Martonyi for photographic services, Ms. J. Smith for typing, and the staff of the Statistical Research Laboratory for the use of the MIDAS Program.

\title{
References
}

Charman, W. N., Tucker, J.: The optical system of the goldfish eye. Vision Res. 13, 1-8 (1973)

Duntley, S. Q.: Light in the sea. J. opt. Soc. Amer. 58, 214-233 (1963)

Easter, S. S.: Spontaneous eye movements in restrained goldfish. Vision Res. 11, 333-343 (1971)

Easter, S. S.: Pursuit eye movements in goldfish (Carassius auratus). Vision Res. 12, 673-688 (1972)

Easter, S. S., Johns, P. R.: Horizontal compensatory eye movements in goldfish (Carassius auratus). II. A comparison of normal and deafferented animals. J. comp. Physiol. 92, 37-57 (1974)

Harris, A. J.: Eye movements of the dogfish Squalus acanthias L. J. exp. Biol. 43, 107-130 (1965)

Hermann, H. T., Constantine, M.: Eye movements in the goldfish. Vision Res. 11, 313-333 (1971)

Lowenstein, O.: The labyrinth. In: Fish physiology, vol. V, p. 207-241 (ed. Hoar, W. D., and D. J. Randall). New York: Academic Press 1971

Rowley, J. B.: Discrimination of pattern and size in the goldfish Carassius auratus. Genet. Psychol. Monogr. 15, 245-302 (1934)

Walls, G. L.: The evolutionary history of eye movements. Vision Res. 2, 69-80 (1962)

Wartzok, D., Marks, W. B.: Directionally selective visual units recorded in optic tectum of the goldfish. J. Neurophysiol. 36, 588-605 (1973)

\author{
Stephen S. Easter, Jr. \\ Pamela R. Johns \\ Donald Heckenlively \\ Department of Zoology \\ University of Michigan \\ Ann Arbor, Michigan 48104, USA
}

